\title{
SUSPENSION SPECTRA AND HOMOLOGY EQUIVALENCES
}

BY

\author{
NICHOLAS J. KUHN ${ }^{1}$
}

\begin{abstract}
Let $f: \Sigma^{\infty} X \rightarrow \Sigma^{\infty} Y$ be a stable map between two connected spaces, and let $E_{*}$ be a generalized homology theory. We show that if $E_{*}(f)$ is an isomorphism then $E_{*}\left(\Omega^{\infty} f\right): E_{*}(Q X) \rightarrow E_{*}(Q Y)$ is a monomorphism, but possibly not an epimorphism. Applications of this theorem include results of Miller and Snaith concerning the $K$-theory of the Kahn-Priddy map.
\end{abstract}

1. Introduction and main results. If $E_{*}$ is a generalized homology theory, a map $f$ : $X \rightarrow Y$ between either spaces or spectra is called an $E_{*}$-equivalence if $E_{*}(f)$ is an isomorphism. It is an interesting and delicate question to study the behavior of $E_{*}$-equivalences after application of various functors to the spaces, spectra, and maps involved. See, for example, $[\mathbf{1}, \mathbf{4}, \mathbf{5}]$.

In particular, consider the functor $\Omega^{\infty}$, the 0 th space functor from the category of spectra to the category of spaces, right adjoint to the suspension spectrum functor $\Sigma^{\infty}$. A variety of spectral sequences exists in the literature to show that if $f: X \rightarrow Y$ is a map between -1 connected spectra and $\Omega^{\infty} f: \Omega^{\infty} X \rightarrow \Omega^{\infty} Y$ is an $E_{*}$-equivalence, then so is $f$. However, the converse need not be true. For example, letting $H \mathbf{Z} / 2$ denote the mod 2 Eilenberg-Mac Lane spectrum, $K_{*}(\Sigma H \mathbf{Z} / 2)=0$ while $K_{*}\left(\mathbf{R} P^{\infty}\right) \neq 0$. (Here we have adopted the convention that if $X$ is a space, $E_{*}(X)$ denotes the reduced homology of $X$.)

Contrasting with this example is a theorem noted by A. K. Bousfield [4]: if $f$ : $X \rightarrow Y$ is an $E_{*}$-equivalence of spaces, so is $Q f: Q X \rightarrow Q Y$, where $Q=\Omega^{\infty} \Sigma^{\infty}$. This suggests that $\Omega^{\infty}$ might be better behaved when restricted to the category of suspension spectra and stable maps. Our main theorem is the following curious extension of Bousfield's result.

THEOREM 1.1. Let $X$ and $Y$ be connected spaces. If $f: \Sigma^{\infty} X \rightarrow \Sigma^{\infty} Y$ is an $E_{*}$-equivalence then $E_{*}\left(\Omega^{\infty} f\right): E_{*}(Q X) \rightarrow E_{*}(Q Y)$ is a monomorphism.

Naturally, we give examples showing that $E_{*}\left(\Omega^{\infty} f\right)$ need not be surjective. Closely related to these examples are some applications of Theorem 1.1 that we make in $\S 3$. These include a noncomputational proof (to within "one loop") of the fact, first proved by H. Miller and V. Snaith [14], that the Kahn-Priddy map $Q B \Sigma_{p} \rightarrow Q_{0} S^{0}$ is a $K_{*}$-equivalence, localized at a prime $p$. Using our solution to $\mathrm{G}$. Whitehead's

Received by the editors June 2, 1983.

1980 Mathematics Subject Classification. Primary 55P42, 55P60; Secondary 55N20, 55P47.

${ }^{1}$ This research was partially supported by NSF grant MCS-8201652 and an A.M.S. Postdoctoral Fellowship. 
symmetric product of spheres conjecture [9] and computations by P. Welcher involving higher Morava $K$-theories [20], we generalize Miller's and Snaith's result. We also show that if the map $\Sigma^{\infty} B \Sigma_{p} \rightarrow \Sigma^{\infty} S^{0}$ is an $E_{*}$-equivalence then $E_{*}\left(K\left(\mathbf{Z}_{(p)}, 2 p^{2}-2\right)\right)=0$. This is interpreted as an inequality between two Bousfield localization classes [2].

The hypothesis that $X$ and $Y$ be connected in Theorem 1.1 is possibly not necessary and appears because of our method of proof. This involves using the stable splitting, valid for connected spaces $X$, due to D. Kahn [7] and Snaith [18]:

$$
\Sigma^{\infty} Q X \simeq \underset{q \geqslant 1}{\vee} \Sigma^{\infty} D_{q} X
$$

Here $D_{q} X$ is the $q$ th extended power construction on $X$. We make use of work by $\mathrm{G}$. Lewis, J. P. May, et al. [11] showing that this construction can also be made in the category of spectra. Indeed, the key to our proof of Theorem 1.1 is a comparison of the maps $\Sigma^{\infty} \Omega^{\infty} f$ and $\vee_{q \geqslant 1} D_{q} f$.

This comparison allows us to extend the Kahn-Snaith splitting theorem to a theorem about "spacelike" spectra, i.e. wedge summands of suspension spectra. These spectra were featured prominently in [10].

Theorem 1.2. Let $P$ be a connected spectrum. Then $P$ is spacelike if and only if there is an equivalence

$$
\Sigma^{\infty} \Omega^{\infty} P \simeq \underset{q \geqslant 1}{\bigvee} D_{q} P
$$

The proofs of Theorems 1.1 and 1.2 , together with related results, comprise $\S \S 2$ and 4.

I would like to thank Pete Bousfield for suggesting improvements in $\S 3$.

2. Proofs of the main theorems. We recall some work basic to understanding the stable splitting of $Q X$. In [12], J. P. May constructed an $E_{\infty}$-space $C X$ together with a natural map $\eta: C X \rightarrow Q X$ preserving all additive structure. This map, furthermore, is a weak equivalence if $X$ is connected and a group completion in general $[12,17]$. $C X$ has a natural increasing filtration and the quotients of successive filtrations are the extended power spaces

$$
D_{q} X=E \Sigma_{q}^{+} \wedge \Sigma_{q} X^{[q]}
$$

Here $E \Sigma_{q}^{+}$is the disjoint union of a basepoint and a contractible space acted on freely by the permutation group $\Sigma_{q}$, and $X^{[q]}$ denotes $X$ smashed with itself $q$ times.

The constructions $D_{q}, q \geqslant 1$, are functors defined on the category of topological spaces. Lewis, May, and M. Steinberger showed that these functors extend to the category of spectra in the following way.

Proposition $2.1[11,13]$. There are functors $D_{q}, q \geqslant 1$, taking spectra to spectra and natural equivalences $D_{q} \Sigma^{\infty} X \simeq \Sigma^{\infty} D_{q} X$.

By filtering $E \Sigma_{q}$ by finite skeleta one can construct the following useful spectral sequence. 
Proposition $2.2[11,13]$. Let $E_{*}$ be a homology theory and $X$ a space or spectrum. There is a spectral sequence, natural in $X$, converging to $E_{*}\left(D_{q} X\right)$ with $E_{* * *}^{2} \simeq$ $H_{*}\left(\Sigma_{q} ; E_{*}\left(X^{[q]}\right)\right)$.

COROllaRY 2.3. Let $f: X \rightarrow Y$ be a map of either spaces or spectra. If $f$ is an $E_{*}$-equivalence, so is $D_{q} f: D_{q} X \rightarrow D_{q} Y$.

As an application of these ideas and to motivate our proof of Theorem 1.1, we give a simple proof of the result of Bousfield mentioned in the introduction.

Proposition 2.4 [4, Remark following Proposition 1.2]. If a map between spaces $f$ : $X \rightarrow Y$ is an $E_{*}$-equivalence so is $Q f: Q X \rightarrow Q Y$.

Proof. There is a commutative diagram of $H$-spaces

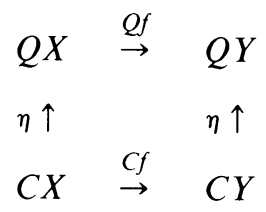

so by the group completion property of $\eta$ it suffices to show that $C f$ is an $E_{*}$-equivalence. This follows immediately from Corollary 2.3 and the diagram

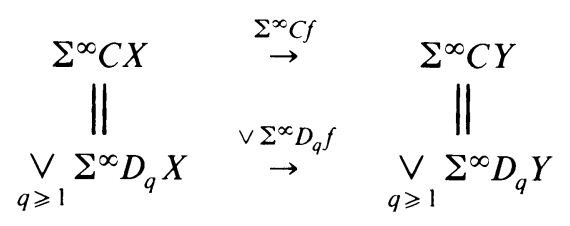

which commutes by the naturality of the Kahn-Snaith splitting.

Theorem 1.1 was discovered by trying to allow $f$ to be a stable map in the above proof. Thus now let $f: \Sigma^{\infty} X \rightarrow \Sigma^{\infty} Y$ be a map of suspension spectra.

A first problem in generalizing the above proof arises if $Y$ is not connected. Then the map $C X \rightarrow Q X \rightarrow \underset{\Omega^{\infty} f}{\rightarrow} Q Y$ need not factor through $C Y \rightarrow Q Y$. For example, if $X=Q Y$ and $f=\varepsilon_{Y}: \Sigma^{\infty} Q Y \rightarrow \Sigma^{\infty} Y$ is the natural evaluation map adjoint to the identity, then the composite

$$
X \rightarrow C X \rightarrow Q X \stackrel{\Omega^{\infty} \varepsilon_{Y}}{\rightarrow} Q Y
$$

is the identity map. Because of examples like this, we now assume $X$ and $Y$ are both connected and, by abuse of notation, identify $Q X$ with $C X, Q Y$ with $C Y$, etc.

A new problem now arises. The diagram

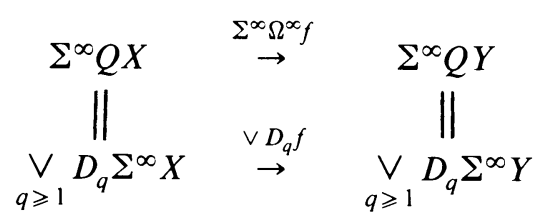


need not commute. For if it did, the composite

$$
\Sigma^{\infty} X \rightarrow \Sigma^{\infty} Q X \stackrel{\Sigma^{\infty} \Omega^{\infty} f}{\rightarrow} \Sigma^{\infty} Q Y \rightarrow D_{q} \Sigma^{\infty} Y
$$

would be null homotopic for all $q>1$, where the unlabeled maps are the natural inclusions or projections. But this is not true for any $q$ if, as in the example above, $X=Q Y$ and $f=\varepsilon_{Y}$.

In spite of this last example, our key geometric observation is that this diagram does, in fact, commute up to the natural decreasing filtration.

THEOREM 2.5. Let $f: \Sigma^{\infty} X \rightarrow \Sigma^{\infty} Y$ be a stable map between two connected spaces. Then the composite

$$
\Sigma^{\infty} D_{p} X \rightarrow \Sigma^{\infty} Q X \stackrel{\Sigma^{\infty} \Omega^{\infty} f}{\rightarrow} \Sigma^{\infty} Q Y \rightarrow \Sigma^{\infty} D_{q} Y
$$

is $D_{p} f$ if $p=q$ and is null homotopic if $q<p$.

Proof. In the spirit of our previous examples, we first note that $\Sigma^{\infty} \Omega^{\infty} f$ can always be factored as the composite

$$
Q X \stackrel{\Sigma^{\infty} Q f^{\prime}}{\rightarrow} \Sigma^{\infty} Q Q Y \stackrel{\Sigma^{\infty} \Omega^{\infty} \varepsilon_{Y}}{\rightarrow} \Sigma^{\infty} Q Y
$$

where $f^{\prime}: X \rightarrow Q Y$ is adjoint to $f$. As in the proof of Proposition 2.4, the naturality of the Kahn-Snaith splitting then reduces the theorem to the case in which $X=Q Y$ and $f=\varepsilon_{Y}: \Sigma^{\infty} Q Y \rightarrow \Sigma^{\infty} Y$.

Thus we study how the map $\Sigma^{\infty} \Omega^{\infty} \varepsilon_{Y}: \Sigma^{\infty} Q Q Y \rightarrow \Sigma^{\infty} Q Y$ relates to the stable splittings. To do this, it is convenient to establish the following notation. Let $D$ be the functor $\vee_{q \geqslant 1} D_{q}$ on the category of either spaces or spectra and note that $\Sigma^{\infty} D Y \simeq D \Sigma^{\infty} Y$. Recall that $D_{q}\left(Y_{1} \vee Y_{2} \vee \cdots\right)$ has a natural decomposition into the wedge summands $D_{q_{1}} Y_{i_{1}} \wedge D_{q_{2}} Y_{i_{2}} \wedge \cdots \wedge D_{q_{n}} Y_{i_{n}}$ with $q_{1}+\cdots+q_{n}=q$ and $i_{1}<i_{2}<\cdots<i_{n}[13,16]$. The group inclusions

$$
\Sigma_{p} \int \Sigma_{q} \rightarrow \Sigma_{p q} \text { and } \quad \Sigma_{p} \times \Sigma_{q} \rightarrow \Sigma_{p+q}
$$

induce maps

$$
D_{p} D_{q} Y \rightarrow D_{p q} Y \quad \text { and } \quad D_{p} Y \wedge D_{q} Y \rightarrow D_{p+q} Y
$$

which in turn define a natural map $\mu: D D Y \rightarrow D Y$.

Let $\pi: D Y \rightarrow Y$ be the projection onto the first component, and, if $Y$ is connected, let $f_{Y}: \Sigma^{\infty} Q Y \rightarrow \Sigma^{\infty} D Y$ be the stable equivalence.

LEMMA 2.6. The following diagrams commute.

(a)

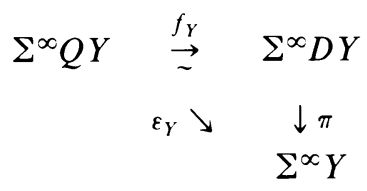


(b)

$$
\begin{array}{ccccc}
\Sigma^{\infty} Q Q Y & & \stackrel{\Sigma^{\infty} \Omega^{\infty} \varepsilon_{Y}}{\rightarrow} & & \Sigma^{\infty} Q Y \\
f_{Q Y} \downarrow & & & & f_{Y} \downarrow \\
\Sigma^{\infty} D Q Y & \stackrel{D f_{Y}}{\sim} & \Sigma^{\infty} D D Y & \stackrel{\mu}{\rightarrow} & \Sigma^{\infty} D Y
\end{array}
$$

Diagram (a) is well known to commute. The commutativity of diagram (b) will be shown in $\S 4$.

We return to the proof of Theorem 2.5. Application of the functor $D$ to diagram (a) of the lemma yields a commutative diagram

$$
\begin{array}{ccc}
\Sigma^{\infty} D Q Y & \stackrel{D f_{Y}}{\rightarrow} & \Sigma^{\infty} D D Y \\
& D \varepsilon_{Y} \downarrow & \downarrow D \pi \\
& & \Sigma^{\infty} D Y
\end{array}
$$

This diagram and diagram (b) of the lemma reduce the theorem to the statement that the composite

$$
D_{p} D Y \rightarrow D D Y \stackrel{\mu}{\rightarrow} D Y \rightarrow D_{q} Y
$$

is $D_{p} \pi$ if $p=q$ and null homotopic if $q<p$. This is clear, because a wedge summand of $D_{p} D Y, D_{p_{1}} D_{i_{1}} Y \wedge \cdots \wedge D_{p_{n}} D_{i_{n}} Y$ with $p_{1}+\cdots+p_{n}=p$ and $i_{1}<\cdots<i_{n}$, will map under $\mu$ to $D_{q} Y$ where $q=p_{1} i_{1}+\cdots+p_{n} i_{n}$.

Proof of Theorem 1.1. Suppose $f: \Sigma^{\infty} X \rightarrow \Sigma^{\infty} Y$ is an $E_{*}$-equivalence between the suspension spectra of two connected spaces. Theorem 2.5 and Corollary 2.3 then immediately imply that, for all finite $n$, the composite

$$
E_{*}\left(\bigvee_{q=1}^{n} \Sigma^{\infty} D_{q} X\right) \rightarrow E_{*}\left(\Sigma^{\infty} Q X\right) \stackrel{E_{*}\left(\Sigma^{\infty} \Omega^{\infty} f\right)}{\rightarrow} E_{*}\left(\Sigma^{\infty} Q Y\right) \rightarrow E_{*}\left(\bigvee_{q=1}^{n} \Sigma^{\infty} D_{q} Y\right)
$$

is an isomorphism. Since $E_{*}(Q X)=\lim _{n \rightarrow \infty} E_{*}\left(\vee_{q \geqslant 1}^{n} D_{q} X\right)$, it follows that $E_{*}\left(\Omega^{\infty} f\right)$ is a monomorphism.

EXAMPLE 2.7. Let $f: \Sigma^{\infty} \Sigma \mathbf{R} P^{\infty} \rightarrow \Sigma^{\infty} S^{1}$ be the Kahn-Priddy map, i.e. let $f$ be any map nonzero on $\pi_{2}$. It is easily checked that $f$ is a $K \mathbf{Z} / 2_{*}$-equivalence, where $K \mathbf{Z} / 2$ is $\bmod 2 \mathrm{~K}$-theory. Hence by Theorem 1.1,

$$
\Omega^{\infty} f_{*}: K_{*}\left(Q \Sigma \mathbf{R} P^{\infty} ; \mathbf{Z} / 2\right) \rightarrow K_{*}\left(Q S^{1} ; \mathbf{Z} / 2\right)
$$

is a monomorphism. However, this is not an epimorphism because under the product splitting $Q S^{1}=Q S^{1}\langle 1\rangle \times S^{1}, f$ factors through $Q S^{1}\langle 1\rangle \rightarrow Q S^{1}$ and thus "misses" $S^{1}$, while $K_{*}\left(S^{1} ; \mathbf{Z} / 2\right) \neq 0$. We will return to this example in $\S 3$.

REMARK 2.8. If $E$ is connective, then the monomorphism of Theorem 1.1 will actually be an isomorphism. Indeed, this follows from our proof together with the fact that $D_{q} X$ becomes increasingly connected as $q$ gets large. In this case, however, Bousfield $[1,3]$ has shown the $E_{*}$-equivalences are the same as $H G_{*}$-equivalences, where $G$ depends on $\pi_{*}(E)$. Thus our theorem is mainly of interest when $\pi_{-i}(E) \neq 0$ for arbitrarily large values of $i$. 
EXAMPLE 2.9. For completeness we note a simple example of a map $f: X \rightarrow Y$ that is $E_{*}$-monic, while $Q f: Q X \rightarrow Q Y$ is not. Let $E=H \mathbf{Z}$, ordinary integral homology, and let 2: $S^{1} \rightarrow S^{1}$ be the degree two map. Then $Q(2)_{*}: H_{*}\left(Q S^{1}\right) \rightarrow H_{*}\left(Q S^{1}\right)$ has nonzero kernel, e.g. in dimension 2 .

Theorem 1.1 implies the next corollary.

Corollary 2.10. If $P$ is a connected spacelike spectrum and $E_{*}(P)=0$ then $E_{*}\left(\Omega^{\infty} P\right)=0$.

Proof. By assumption there is a space $X$ and spectrum $P^{\prime}$ such that $P \vee P^{\prime}=$ $\Sigma^{\infty} X$. We can also assume $X$ is connected, since $X$ could be $\Omega^{\infty} P$ in particular (see [10]). Consider the composite

$$
E_{*}\left(\Omega^{\infty} P\right) \stackrel{f}{\rightarrow} E_{*}(Q X) \stackrel{g}{\rightarrow} E_{*}\left(\Omega^{\infty} P^{\prime}\right) \stackrel{h}{\rightarrow} E_{*}(Q X) .
$$

The map $f$ must be monic and $g f$ must be zero. By the theorem, $h g$, and thus $g$, must be monic. The corollary follows.

Proof of Theorem 1.2. Suppose $P \vee P^{\prime}=\Sigma^{\infty} X$ and $X$ is connected. We need to show $\Sigma^{\infty} \Omega^{\infty} P=\vee_{q \geqslant 1} D_{q} P$. As in the proof of Theorem 2.5 , we let $D P=\vee_{q \geqslant 1} D_{q} P$. Let $f: D P \rightarrow \Sigma^{\infty} \Omega^{\infty} P$ be the composite

$$
D P \rightarrow \Sigma^{\infty} D X \rightarrow \Sigma^{\infty} Q X \rightarrow \Sigma^{\infty} \Omega^{\infty} P .
$$

Similarly, one can define $g: D P^{\prime} \rightarrow \Sigma^{\infty} \Omega^{\infty} P^{\prime}$. Note that

$$
\Sigma^{\infty} Q X=\Sigma^{\infty} \Omega^{\infty} P \vee \Sigma^{\infty} \Omega^{\infty} P^{\prime} \vee\left(\Sigma^{\infty} \Omega^{\infty} P \wedge \Sigma^{\infty} \Omega^{\infty} P^{\prime}\right)
$$

and

$$
\Sigma^{\infty} D X=D P \vee D P^{\prime} \vee\left(D P \wedge D P^{\prime}\right) .
$$

Under these identifications, let

$$
h=f \vee g \vee(f \wedge g): \Sigma^{\infty} D X \rightarrow \Sigma^{\infty} Q X .
$$

By Theorem 2.5 applied to the maps $\Sigma^{\infty} X \rightarrow P \rightarrow \Sigma^{\infty} X$ and $\Sigma^{\infty} X \rightarrow P^{\prime} \rightarrow \Sigma^{\infty} X$, the composite

$$
\Sigma^{\infty} D X \stackrel{h}{\rightarrow} \Sigma^{\infty} Q X \underset{\sim}{\sim} \Sigma^{\infty} D X
$$

is the identity map up to filtration. Since $D_{q} X$ becomes highly connected for large $q$, $h$ must be an isomorphism on $\pi_{*}$, thus an equivalence. We conclude that both $f$ and $g$ are equivalences.

Theorem 1.2 allows us to extend Theorem 1.1 to maps between spacelike spectra, strengthening Corollary 2.10.

Corollary 2.11. If $f: P \rightarrow P^{\prime}$ is an $E_{*}$-equivalence between connected spacelike spectra, then $E_{*}\left(\Omega^{\infty} f\right)$ is a monomorphism.

Proof. Theorem 1.2 allows us to identify $\Sigma^{\infty} \Omega^{\infty} P$ with $D P, \Sigma^{\infty} \Omega^{\infty} P^{\prime}$ with $D P^{\prime}$, and to regard $D f$ as a map $\Sigma^{\infty} \Omega^{\infty} P \rightarrow \Sigma^{\infty} \Omega^{\infty} P^{\prime}$. By Corollary 2.3 this map is an 
$E_{*}$-equivalence. There is a commutative diagram

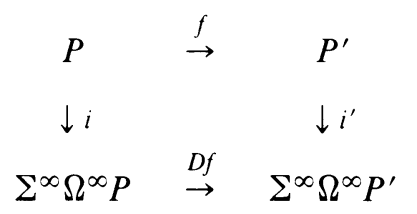

and the fact that $\Omega^{\infty} f$ is an $E_{*}$-monomorphism then follows from the fact that $\Omega^{\infty} i$ and $\Omega^{\infty} D f$ are also $E_{*}$-monic; $\Omega^{\infty} D f$ by use of Theorem 1.1.

3. Applications. As a first application of Theorem 1.1, let $\lambda: \Sigma^{\infty} B \Sigma_{p} \rightarrow \Sigma^{\infty} S^{0}$ be any map that is nonzero on $p$ torsion in $\pi_{2 p-3}$. There is a factorization $\Sigma^{\infty} B \Sigma_{p} \stackrel{\lambda^{\prime}}{\rightarrow} S\langle 0\rangle \rightarrow \Sigma^{\infty} S^{0}$ of $\lambda$ through the zero connected cover of $\Sigma^{\infty} S^{0}$. As in Example 2.7, let $Q S^{1}\langle 1\rangle$ be the simply connected cover of $Q S^{1}$.

THEOREM 3.1. Localized at $p$, if $\lambda$ is an $E_{*}$-equivalence, so is $\Omega^{\infty} \Sigma \lambda^{\prime}: Q \Sigma B \Sigma_{p} \rightarrow$ $Q S^{\mathrm{l}}\langle 1\rangle$.

Proof. By Theorem 1.1, $\Omega^{\infty} \Sigma \lambda$, and thus $\Omega^{\infty} \Sigma \lambda^{\prime}$, is an $E_{*}$-monomorphism. But by the "delooped" Kahn-Priddy Theorem [8], localized at $p, Q S^{1}\langle 1\rangle$ is a retract of $Q \Sigma B \Sigma_{p}$ via $\Omega^{\infty} \Sigma \lambda^{\prime}$. Thus $\Omega^{\infty} \Sigma \lambda^{\prime}$ must be an $E_{*}$-epimorphism.

COROLlary 3.2 [14]. Localized at $p, \Omega^{\infty} \Sigma \lambda^{\prime}: Q \Sigma B \Sigma_{p} \rightarrow Q S^{1}\langle 1\rangle$ is a $K_{*}$-equivalence.

Proof. As observed by Miller and Snaith, it suffices to show that $\Omega^{\infty} \Sigma \lambda^{\prime}$ is a $K \mathbf{Z} / p_{*}$-equivalence. By a simple Atiyah-Hirzebruch spectral sequence calculation, the cofiber of $\lambda$ is $K \mathbf{Z} / p_{*}$-acyclic. Thus $\lambda$ is a $K \mathbf{Z} / p_{*}$-equivalence and we can apply Theorem 3.1.

Note that we have missed the full strength of Miller's and Snaith's result by "one loop". The stronger theorem can also be obtained by using Bousfield's quite elegant characterization of $K_{*}$-equivalences between infinite loop spaces [4]. However, these other approaches involve much more computational information about $K$-theory.

Using calculations by P. Welcher, we now recover the stronger version of Corollary 3.2 and generalize it for $p=2$. Localizing at a prime $p$, recall that $H \mathbf{Z}$ is filtered by the symmetric product spectra $S P^{p^{n}}(S)$. Let

$$
E_{n}=\Sigma^{-n-1} H \mathbf{Z} / S P^{p^{n}}(S) \text { and } L(n)=\Sigma^{-n} S P^{p^{n}}(S) / S P^{p^{n-1}}(S) .
$$

Note that there are cofibrations $E_{n} \rightarrow L(n) \rightarrow E_{n-1}$ and identifications $L(1)=$ $\Sigma^{\infty} B \Sigma_{p}, E_{0}=S\langle 0\rangle[9,19]$. Let $K(m)_{*}$ be the $m$ th Morava $K$-theory.

THEOREM 3.3. Localized at $p=2, \Omega^{\infty} L(n) \rightarrow \Omega^{\infty} E_{n-1}$ is a $K(n)_{*}$-equivalence $e_{*}$ If $m<n$,

$$
K(m)_{*}\left(\Omega^{\infty} L(n)\right)=K_{*}(m)_{*}\left(\Omega^{\infty} E_{n-1}\right)=0 .
$$

Proof. Calculations by Welcher in [20] imply $K(m)_{*}(L(n))=0$ if $m<n$. Priddy and Mitchell show that $L(n)$ is spacelike in [15], so Corollary 2.10 implies 
$K(m)_{*}\left(\Omega^{\infty} L(n)\right)=0$ if $m<n$. The remaining assertions in the theorem then follow from the fact that $\Omega^{\infty} L(n)=\Omega^{\infty} E_{n} \times \Omega^{\infty} E_{n-1}$, the main result of [9].

To state our next results, we recall Bousfield's notion of localization classes of spectra [2]. The homotopy category of C.W. spectra is partially ordered by writing $A \geqslant B$ if $E_{*}(B)=0$ whenever $E_{*}(A)=0$. This partial ordering partitions spectra into equivalence classes with the equivalence class of a spectrum $A$ denoted by $\langle A\rangle$. There are well-defined operations $\vee$ and $\wedge$ defined on equivalence classes in the obvious way.

THEOREM 3.4. Localized at a prime $p$

$\left\langle S P^{p}(S)\right\rangle \geqslant\left\langle\Sigma^{\infty} K\left(\mathbf{Z} / p, 2 p^{2}-3\right)\right\rangle$ and $\left\langle S p^{p}(S)\right\rangle \geqslant\left\langle\Sigma^{\infty} K\left(\mathbf{Z}_{(p)}, 2 p^{2}-2\right)\right\rangle$.

Equivalently, if the Kahn-Priddy map $\lambda: \Sigma^{\infty} B \Sigma_{p} \rightarrow \Sigma^{\infty} S^{0}$ is an $E_{*}$-equivalence then

$$
E_{*}\left(K\left(\mathbf{Z} / p, 2 p^{2}-3\right)\right)=E_{*}\left(K\left(\mathbf{Z}_{(p)}, 2 p^{2}-2\right)\right)=0 .
$$

Proof. First note that the cofiber of $\lambda$ is $S P^{p}(S)$ [19]. If $\lambda$ is an $E_{*}$-equivalence then, by Theorem 3.1, $\Omega^{\infty} \Sigma \lambda^{\prime}: Q \Sigma B \Sigma_{p} \rightarrow Q S^{1}\langle 1\rangle$ is an $E_{*}$-equivalence. The smallest $n$ for which

$$
\left(\Omega^{\infty} \Sigma \lambda^{\prime}\right)_{*}: H_{n}\left(Q \Sigma B \Sigma_{p} ; \mathbf{Z} / p\right) \rightarrow H_{n}\left(Q S^{1}\langle 1\rangle ; \mathbf{Z} / p\right)
$$

is not an isomorphism in $n=2 p^{2}-3$. The theorem then follows from Bousfield's results in [5, Proposition 2.1, Lemma 3.4, §4].

Proposition 3.5. (a) If $P$ is spacelike then $\langle P\rangle=\left\langle\Sigma^{\infty} \Omega^{\infty} P\right\rangle$.

(b) If $A \rightarrow B \rightarrow C$ is short exact and $B$ is spacelike then $\langle B\rangle=\langle A\rangle \vee\langle C\rangle$.

Proof. The first assertion is a restatement of Corollary 2.10. To prove (b) it suffices to show $\langle B\rangle \geqslant\langle C\rangle$. This is easy:

$$
E_{*}(B)=0 \Rightarrow E_{*}\left(\Omega^{\infty} B\right)=0 \Rightarrow E_{*}\left(\Omega^{\infty} C\right)=0 \Rightarrow E_{*}(C)=0 .
$$

Corollary 3.6. $\langle L(n)\rangle=\left\langle E_{n}\right\rangle \vee\left\langle E_{n-1}\right\rangle$, localized at $p=2$.

Recall that $S P^{\infty}(S)=H \mathbf{Z}$ by the Dold-Thom Theorem. A simple direct argument or Theorem 3.4 implies that, localized at $\left.p,\left\langle S P^{p}(S)\right\rangle\right\rangle\left\langle S P^{\infty}(S)\right\rangle$. This suggests the following conjecture.

CONJECTURE. $\left.\left\langle S P^{p^{n}}(S)\right\rangle\right\rangle\left\langle S P^{p^{n+1}}(S)\right\rangle$ and $\left.\left\langle E_{n}\right\rangle\right\rangle\left\langle E_{n+1}\right\rangle$.

REMARK 3.7. As a consequence of this conjecture, one would have $\langle L(n)\rangle=$ $\left\langle E_{n-1}\right\rangle$ and thus $\left\langle E_{n}\right\rangle=\left\langle\Sigma^{\infty} \Omega^{\infty} E_{n}\right\rangle$. Related to this, we note that results in [15] easily imply $\langle L(n)\rangle>\langle L(n+1)\rangle$. In work in progress, we have also checked that $\left\langle S P^{p}(S)\right\rangle>\left\langle S P^{p^{n}}(S)\right\rangle$ for $n>1$, which leads to an alternate proof of Theorem 3.4 and avoids use of the Kahn-Priddy Theorem.

4. Proof of Lemma 2.6. In this section we prove the commutativity of diagram (b) of Lemma 2.6. We work solely with the formal properties of various spectrum level constructions made in [11], and our proof is similar in spirit to R. Cohen's "stable proof" of the stable splitting of $Q X[6]$. 
Recall that there is a functor $C$ defined on the category of spectra with unit. This is related to the space level construction via the natural equivalence $C \Sigma^{\infty}\left(X^{+}\right) \simeq$ $\Sigma^{\infty}(C X)^{+}$and is related to $D=\vee_{q \geqslant 1} D_{q}$ via $C(E \vee S) \simeq D(E) \vee S$, where $S$ is the sphere spectrum.

There are natural transformations $\theta: C^{2} \rightarrow C$ and $\eta: 1 \rightarrow C$ making $C$ into a monad. These are related to the space level constructions in the obvious way, and $\theta$ is related to $\mu: D^{2} \rightarrow D$ through the commutative square:

$$
\begin{array}{ccc}
C C(E \vee S) & \simeq D D E \vee S \\
\downarrow \theta & \quad \downarrow \mu \vee 1 \\
C(E \vee S) & \simeq D E \vee S .
\end{array}
$$

LEMMA 4.1. The following diagram commutes, naturally, for all spaces $X$.

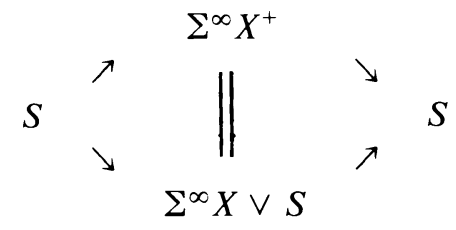

COROllary 4.2. There is natural equivalence $f_{X}: \Sigma^{\infty} C X \rightarrow \Sigma^{\infty} D X$ such that the composite

$$
\Sigma^{\infty} C X \vee S=\Sigma^{\infty}(C X)^{+}=C\left(\Sigma^{\infty} X^{+}\right)=C\left(\Sigma^{\infty} X \vee S\right)=\Sigma^{\infty} D X \vee S
$$

is $f_{X} \vee 1$.

Proof. Let the composite be $\left(\begin{array}{ll}f & g \\ h & g\end{array}\right)$ in matrix form. The left triangle of Lemma 4.1 implies $g=0$ and $i=1: S \rightarrow S$. The right triangle implies $h=0$. Let $f_{X}=f$.

If $X$ is a space, the commutativity of the diagram

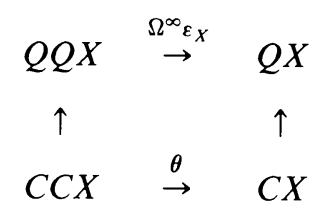

(see [12]) reduces Lemma 2.6 to the next proposition.

Proposition 4.3. There is a naturally commuting diagram:

$$
\begin{array}{ccc}
\Sigma^{\infty} C C X & \stackrel{\Sigma^{\infty} \theta}{\rightarrow} & \Sigma^{\infty} C X \\
f_{C X} \downarrow & & f_{X} \downarrow l \\
\Sigma^{\infty} D C X & \stackrel{D f_{X}}{\rightarrow} \Sigma^{\infty} D D X \stackrel{\mu}{\rightarrow} & \Sigma^{\infty} D X .
\end{array}
$$


Proof. Consider the following diagram

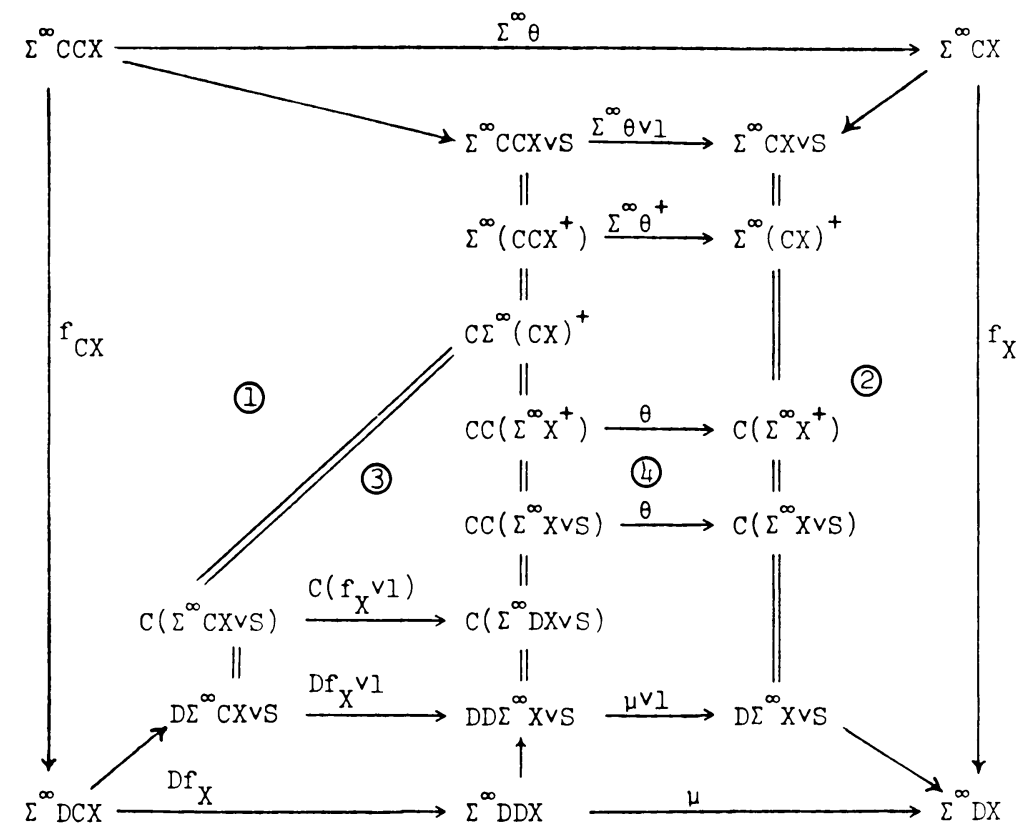

(1) and (2) commute by Corollary 4.2. That (3) commutes can be seen by applying the functor $C$ to the composite of the corollary. (4) commutes by naturality. The proposition follows.

\section{REFERENCES}

1. A. K. Bousfield, Types of acyclicity, J. Pure Appl. Algebra 4 (1974), 293-298.

2. __ The Boolean algebra of spectra, Comment. Math. Helv. 54 (1979), 368-377.

3. W The localization of spectra with respect to homology, Topology 18 (1979), 257-281.

4. K-localizations and K-equivalences of infinite loop spaces, Proc. London Math. Soc. (3) 44 (1982), 291-311.

5. On homology equivalences and homological localizations of spaces, Amer. J. Math. 104 (1982), 1025-1042.

6. R. L. Cohen, Stable proofs of stable splittings, Math. Proc. Cambridge Philos. Soc. 88 (1980), $149-152$.

7. D. S. Kahn, On the stable decomposition of $\Omega^{\infty} S^{\infty} A$, Lecture Notes in Math., vol. 658, Springer-Verlag, Berlin and New York, 1978, pp. 206-213.

8. N. J. Kuhn, The geometry of the James-Hopf maps, Pacific J. Math. 102 (1982), 397-412.

9. , A Kahn-Priddy sequence and a conjecture of G. W. Whitehead, Math. Proc. Cambridge Philos. Soc. 92 (1982), 467-483.

10. Spacelike resolutions of spectra, Proc. Northwestern Homotopy Theory Conf., Contemporary Math., vol. 19, Amer. Math. Soc., Providence, R. I., 1983, pp. 153-165.

11. G. Lewis, J. P. May, J. McClure and M. Steinberger, Equivariant stable homotopy, Chapter 5. Lecture Notes in Math., Springer-Verlag, (to appear).

12. J. P. May, The geometry of iterated loop spaces, Lecture Notes in Math., vol. 271, Springer-Verlag, Berlin and New York, 1972.

13. , $H_{\infty}$ ring spectra and their applications, Algebraic and Geometric Topology, Proc. Sympos. Pure Math., vol. 32, part 2, Amer. Math. Soc., Providence, R.I., 1978, pp. 229-243. 
14. H. Miller and V. P. Snaith, On the K-theory of the Kahn-Priddy map, J. London Math. Soc. 20 (1979), 339-342.

15. S. A. Mitchell and S. B. Priddy, Stable splittings derived from the Steinberg module, Topology 22 (1983), 285-298.

16. G. Nishida, The nilpotency of elements of the stable homotopy groups of spheres, J. Math. Soc. Japan 25 (1973), 707-732.

17. G. Segal, Configuration spaces and iterated loop spaces, Invent. Math. 21 (1973), 213-221.

18. V. P. Snaith, $A$ stable decomposition for $\Omega^{n} S^{n} X$, J. London Math. Soc. 7 (1974), 577-583.

19. P. J. Welcher, Symmetric products and the stble Hurewitz homomorphism, Illinois J. Math. 24 (1980), $527-544$.

20. __ Symmetric fiber spectra and K(n)-homology acyclicity, Indiana J. Math. 30 (1981), 801-812.

Department of Mathematics, Princeton University, Princeton, New Jersey 08544 